Copyright (C) [2006] IEEE. Reprinted from (Special Issue on Nonlocal, Collisionless Electron Transport in Plasmas - June 2006) .

This material is posted here with permission of the IEEE. Internal or personal use of this material is permitted. However, permission to reprint/republish this material for advertising or promotional purposes or for creating new collective works for resale or redistribution must be obtained from the IEEE by writing to pubs-permissions@ieee.org.

By choosing to view this document, you agree to all provisions of the copyright laws protecting it. 


\section{Energy Balance and Plasma Potential in Low-Density Hot-Filament Discharges}

Scott Robertson, Scott Knappmiller, and Zoltan Sternovsky

Abstract-Electron energy balance is shown to play an important role in determining the plasma potential in low-density hot-filament discharges. The confined electrons that are lost to the walls are those with energy just above the plasma potential, thus the electron energy loss rate is the product of the electron loss rate and the height of the potential barrier. The sources of the electron energy are the energy at creation plus the energy gained from equilibration with energetic, unconfined electrons. An experiment in a souppot plasma device demonstrates that the plasma potential has values that satisfy the energy balance equation. The ion loss rate affects the electron loss rate through the quasineutrality condition, thus collisions of ions play a role in determining the plasma potential by reducing the particle loss rates.

Index Terms_-Plasma devices, plasma measurements, plasma sheaths

S. Robertson and S. Knappmiller are with the Center for Integrated Plasma Studies and the Department of Physics, University of Colorado, Boulder, CO 80304-0390 USA. (email: scott.robertson@colorado.edu).

Z. Sternovsky is with the Laboratory of Atmospheric and Space Physics, University of Colorado, Boulder, CO 80304-392 USA. 


\section{INTRODUCTION}

A recent model for particle balance and energy balance in low-density, hotfilament discharges ${ }^{1}$ gives values for electron temperature and plasma potential that agree (to within about $25 \%$ ) with values measured in a soup-pot type ${ }^{2}$ of plasma device operated at neutral gas pressures of 0.1-1 mTorr. In this work, the energy balance model is extended to higher pressure ( $8 \mathrm{mTorr}$ ) by including the effect of charge-exchange collisions of the ions. An important dimensionless parameter is the ratio of the plasma radius $r$ to the charge-exchange mean free path $\lambda$. At the higher pressure of 8 mTorr, $r / \lambda$ $\cong 30$ and charge-exchange collisions significantly reduce the ion loss rate. The model with collisions is in agreement with experimental data at higher pressure.

The energy balance model can be summarized as follows. The confined electrons are those with energy below the plasma potential $\Phi_{p}$. Electrons are lost from the confined population by diffusion in velocity that allows some of the confined electrons to pass over the potential barrier. If there are no other significant energy loss mechanisms, the rate at which the population of confined electrons loses energy is simply the product of the electron loss rate and $q \Phi_{p}$ where $q$ is the elementary charge. In a steady state, this energy loss is balanced by two sources. The first is the equilibration of the confined electrons with the more energetic unconfined electrons that include the primary electrons as well as the more numerous secondary electrons from the walls. ${ }^{3,4}$ The second energy source is the energy with which the electrons are created. This work and ref. [1] builds upon earlier work ${ }^{3,4,5,6}$ that enumerated the processes affecting energy balance in soup- 
pot types of devices. These devices have not previously been modeled with the level of detail that has been used for DC discharges at higher pressure. ${ }^{7,8,9}$

Charge-exchange collisions replace ions that have been accelerated toward the wall with ions that move more slowly, thus the ion loss rate is decreased by collisions. The electron and ion loss rates are coupled by quasineutrality, thus an effect of ionneutral collisions is a reduction in both the ion and electron loss rates. A reduction in the electron loss rate results in the electrons gaining more energy through equilibration and thus a greater plasma potential (or a greater electron loss rate) is required to satisfy energy balance. We observe in the experiment an increase in plasma potential with neutral gas pressure. This increase is approximately equal to that predicted by the energybalance model when the effect of charge-exchange collisions is included.

In Sec. II, the model for electron energy balance is developed. The rate of ion loss to the wall is derived, including the effect of collisions, and an expression for energy balance is derived from which the plasma potential can be calculated. In Sec. III, the experiment is described and the measured plasma potentials are compared with those calculated using the energy balance model. Section IV is a conclusion.

\section{THE ENERGY BALANCE MODEL}

\section{A. Ion production and loss}

Electron-ion pairs are assumed to be created at the rate $\widehat{R} V$ where $\widehat{R}$ is the volume-average of the rate of ionization and $V$ is the plasma volume. The loss rate of ions 
is $\Gamma_{\mathrm{i}} A$, where $\Gamma_{\mathrm{i}}$ is the flux of ions to the walls and $A$ is the wall area. The volumeaveraged rate of ionization may then be found from

$$
\widehat{R}=\frac{\Gamma_{i} A}{V} .
$$

In the absence of collisions, the flux of ions to the walls in cylindrical geometry is $\sim 0.42$ $n_{e} c_{\mathrm{s}}$, where $n_{e}$ is the electron density, $c_{s}=\sqrt{T_{e} / m_{i}}$ is the ion sound speed, $T_{e}$ is the temperature of confined electrons in energy units, and $m_{i}$ is the ion mass. ${ }^{10,11}$ This ion

flux is reduced by charge-exchange collisions. ${ }^{12,13}$ Sternovsky ${ }^{14}$ has used a kinetic model to calculate $\Gamma_{\mathrm{i}}$ for a range of $r / \lambda$, where $r$ is the plasma radius and $\lambda$ is the chargeexchange mean free path. The numerical results for $r / \lambda<1000$ can be fit to the function

$$
\Gamma_{i}=\frac{0.42 n_{e} c_{s}}{\sqrt{1+0.18(r / \lambda)}},
$$

and the ionization rate can then be found from more easily measured quantities using

$$
\hat{R}\left(n_{e}, T_{e}, \lambda\right)=\frac{0.42 n_{e} A \sqrt{T_{e} / m_{i}}}{V \sqrt{1+0.18(r / \lambda)}} .
$$

\section{B. Electrons from ionization}

Only a fraction of the electrons from electron impact ionization have energy sufficiently low to be confined by the plasma potential. The distribution in energy $S(E)$ of 
secondary electrons from ionization, Fig. 1A, has been measured for several gases by Opal et al. ${ }^{15}$ and is approximately

$$
S(E) \cong \frac{2}{\pi W}\left(\frac{1}{1+(E / W)^{2}}\right)
$$

where $E$ is the energy of the secondary electron and $W=10 \mathrm{eV}$ for Ar. A more accurate normalization to unity can be made by cutting off the distribution at the maximum energy of a secondary electron. This energy is $P=\left(E_{p r i}-E_{i}\right) / 2$, where $E_{p r i}$ is the energy of the primary electrons from the filaments and $E_{i}$ is the ionization energy. The fraction $F\left(\Phi_{p}\right)$

of the source distribution that is confined by the plasma potential is then ${ }^{16}$

$$
F\left(\Phi_{p}\right)=\frac{\arctan \left(q \Phi_{p} / W\right)}{\arctan (P / W)} \cong \frac{q \Phi_{p}}{W \arctan (P / W)}
$$

The final expression is valid for $q \Phi_{p}<<W$. The rate at which confined electrons are created and lost in a steady state is $\widehat{R}\left(n_{e}, T_{e}, \lambda\right) F\left(\Phi_{p}\right)$.

\section{Electron heating from equilibration with secondaries}

Figure 1B illustrates the electron distribution function that is found from probe measurements. ${ }^{17}$ The electrons with energy below $q \Phi_{p}$ are typically confined for many electron-electron collision times, thus this part of the electron distribution is nearly Maxwellian. Ionization is from energetic primary electrons which usually have energy in 
the range 40-100 eV. The primaries release secondary electrons from the walls that have a distribution in energy that is approximately Maxwellian with a temperature of $T_{s e} \approx 2$ $\mathrm{eV}$. The secondary electrons from the wall are accelerated through the sheath potential and are thus shifted upward in energy by $q \Phi_{p} .{ }^{3,4}$ The distribution in velocity of wall secondaries within the plasma is then

$$
\begin{array}{rlr}
f_{s e}(v) & =n_{s e}\left(\frac{m_{e}}{2 \pi T_{s e}}\right)^{\frac{3}{2}} \exp \left[\frac{-0.5 m_{e} v^{2}+q \Phi_{p}}{T_{s e}}\right] & v^{2} \geq 2 q \Phi_{p} / m_{e} \\
& =0, & v^{2}<2 q \Phi_{p} / m_{e}
\end{array}
$$

where $n_{s e}$ is the number density of wall secondaries that would be observed at the wall. This density is found consistently from the probe data using the random current of secondaries collected by the probe when it is at zero potential relative to the wall.

The rate at which a single energetic electron transfers energy to the confined electrons is ${ }^{18,19}$

$$
\frac{d U}{d t}=\frac{Y n_{e} m_{e}}{v}
$$

where $n_{e}$ is the density of the confined electrons, $v$ is the relative velocity of the collisions, $Y=4 \pi\left(q^{2} / 4 \pi \varepsilon_{0} m_{e}\right)^{2} \ln \Lambda$ and $\ln \Lambda$ is the Coulomb logarithm. The energy 
loss rate can be integrated over the distribution of wall secondaries to find the rate at which energy is transferred from the wall secondaries to the confined population ${ }^{1}$

$$
\begin{aligned}
\frac{d Q}{d t}= & Y n_{e} m_{e}\left\langle\frac{n_{s e}}{v}\right\rangle \\
& =Y n_{e} m_{e} n_{s e}\left(\frac{m_{e}}{2 \pi T_{s e}}\right)^{\frac{3}{2}} \exp \left(q \Phi_{p} / T_{s e}\right) \int_{w}^{\infty} \exp \left(-m_{e} v^{2} / 2 T_{s e}\right) 4 \pi v d v, \\
& =\frac{2 m_{e} Y n_{e} n_{s e}}{\sqrt{2 \pi T_{s e} / m_{e}}}
\end{aligned}
$$

where the angled brackets denote an average over the distribution function of wall secondaries and $w=\sqrt{2 q \Phi_{p} / m_{e}}$ is the minimum velocity of wall secondaries within the plasma. The final result for the heating rate is independent of the potential $\Phi_{p}$ and the minimum velocity of the secondaries $w$ because the factors containing these variables cancel.

\section{Electron energy balance}

The energy balance equation for confined electrons must include both the energy from equilibration $d Q / d t$ and the mean energy that the electrons from ionization have initially. The distribution of initial energy, Eq. (4) and Fig. 1A, is nearly flat from zero energy to $q \Phi_{p}$, thus the mean initial energy is approximately $\frac{1}{2} q \Phi_{p}$. The rates of energy input are set equal to the rate at which energy is carried to the wall to obtain the energy balance relation 


$$
\frac{d Q}{d t}+\frac{1}{2} \hat{R}\left(n_{e}, T_{e}, \lambda\right) F\left(q \Phi_{p}\right) q \Phi_{p}=\hat{R}\left(n_{e}, T_{e}, \lambda\right) F\left(q \Phi_{p}\right) q \Phi_{p},
$$

which can be rearranged to give

$$
\Phi_{p}=\frac{2 \frac{d Q}{d t}}{q \widehat{R}\left(n_{e}, T_{e}, \lambda\right) F\left(q \Phi_{p}\right)} .
$$

Equations (3) and (5) can then be used to find the relationship between the plasma potential and other parameters that can be found from probe data

$$
\Phi_{p}\left(n_{s e}, T_{s e}, T_{e}, \lambda\right)=\frac{1}{q}\left[\frac{9.3 W \arctan (P / W) Y m_{e} n_{s e} V \sqrt{1+0.18 r / \lambda}}{A \sqrt{2 \pi T_{e} T_{s e} / m_{i} m_{e}}}\right]^{1 / 2} .
$$

This equation shows that the plasma potential is not explicitly dependent upon $n_{e}$ or $\hat{R}$ and is only weakly dependent upon $T_{e}$. The strongest dependence is upon the density of secondary electrons which are the source of heating for the confined electrons. The energetic primaries have not been included as a source of heating because their transfer of energy is much smaller as a consequence of their higher velocity and lower density. In ref. [1], three variables $\left(n_{e}, T_{e}\right.$, and $\left.\Phi_{p}\right)$ were treated as unknowns and were found by solving simultaneously ion particle balance, electron particle balance, and electron energy balance. In this work, the electron particle balance equation is omitted and the number of unknowns is reduced by taking $T_{e}$ from probe data. Having a measured 
value for $T_{e}$ rather than a model value for $T_{e}$ removes any questions that might arise about the accuracy of the model value for $T_{e}$.

\section{THE EXPERIMENT}

\section{A. The apparatus}

The experiments are performed in a soup-pot type of plasma device, Fig. 2. The vacuum chamber is of aluminum with an inner diameter of $31 \mathrm{~cm}$ and a length of $70 \mathrm{~cm}$. The chamber has a stainless steel liner to cover contamination and to make the surface potential more uniform. ${ }^{20} \mathrm{~A}$ turbomolecular pump creates the vacuum and the base pressure is $<10^{-6}$ Torr. The plasma is generated by primary electrons from four filaments located on the end walls. The filament bias potential is $-80 \mathrm{~V}$ and the emission current is $20-160 \mathrm{~mA}$. The working gas is argon at pressures of $0.1-8 \mathrm{mTorr}$. The pressure is measured by an ionization gauge with an extended range. The mean free path for scattering of the primary electrons is about $5 \mathrm{~cm}$ at the highest pressure, thus the ionization rate is higher near the filaments than at the center of the chamber. The confined electrons (with energies below $\sim 0.5 \mathrm{eV}$ ) have a mean free path comparable to the chamber diameter, thus these electrons should fill the chamber nearly uniformly except in the sheath region at the walls. Probe measurements indicate that the density of confined electrons at the center of the chamber is about $10 \%$ lower than the density 10 $\mathrm{cm}$ from the end walls.

The plasma parameters are determined by means of a cylindrical probe of stainless steel with a diameter of $190 \mu \mathrm{m}$ and a length of $27 \mathrm{~mm}$. The probe is discharge- 
cleaned before data are taken. A digital data acquisition system with 16-bit resolution averages 25 current readings at each probe voltage. A subset of the probe data from -40 to $-10 \mathrm{~V}$ are fit to a model for the ion current and this current is subtracted from the probe current to obtain the electron current alone, Fig. 3. This current shows two electron distributions: a low energy $(<0.5 \mathrm{eV})$ distribution that is the confined electrons and a higher energy $(>0.5 \mathrm{eV})$ distribution that is the wall secondaries. Orbit-motion-limited theory $y^{21,22}$ is used to find the densities and temperatures of the two populations as described in ref. 17. The electrons contributing to the probe current for voltages from 0 to -2 volts is identified as secondaries from the wall because the slope of the semilogarithmic plot corresponds to the expected temperature of $2-3 \mathrm{eV}$. This part of the probe current cannot be primary electrons because primaries have energy near $80 \mathrm{eV}$ and would create a probe current with a much smaller slope. The density of the wall secondaries is typically a few percent of the total electron density.

The analysis locates the effective wall potential by finding the probe voltage at which the current of confined electrons begins to rise above the current of wall secondaries. This point is typically within 0.1 volt of the ground potential. The plasma potential is located at the maximum in the slope $\mathrm{e}^{23}$ of a function fit to the probe data. The potential $\Phi_{p}$ is found from the difference between the probe voltages at the plasma potential and at the wall potential. These are each uncertain by $0.1 \mathrm{~V}$ as a consequence of the spacing of the data points. These two uncertainties are added in quadrature to obtain the uncertainty of $0.14 \mathrm{~V}$ in $\Phi_{p}$. 


\section{B. The data}

The model is tested by comparing the measured plasma potential with the potential calculated from the model. Parameter scans are made by varying either the filament emission current or the filling pressure. The plasma potential is dependent upon $n_{s e}, T_{s e}$, and $T_{e}$, which are found from the probe data, and upon the mean-free-path $\lambda$. The mean-free-path is determined from $\lambda=1 / n_{n} \sigma$ where $n_{n}$ is the gas density calculated from the pressure gauge reading and $\sigma$ is the charge exchange cross section. This cross section is $72 \times 10^{-16} \mathrm{~cm}^{2}$ for argon ions on argon neutrals. ${ }^{24}$ Figure 4 shows emission-scan data from $20 \mathrm{~mA}$ to $160 \mathrm{~mA}$ at a constant pressure of $0.5 \mathrm{mTorr}$. The top panel shows the measured plasma potential and the plasma potential from the model, Eq. (11). The lower two panels show the data that are the inputs to the model. The mean free path for charge exchange is approximately half the chamber radius, and the collisional decrease in ion flux to the wall [from Eq. (2)] is only $16 \%$. The number density of the secondary electrons responsible for heating is varied by more than a factor of six. The different values of emission change every parameter that enters into Eq. (11) except $\lambda$, and also changes the number density of confined electrons $n_{\mathrm{e}}$. The model for the plasma potential agrees with the data to within the uncertainty in the measurements.

Pressure-scan data from 0.125 to 8 mTorr with a constant emission of $80 \mathrm{~mA}$ are shown in Fig. 5. The range of pressure corresponds to $r / \lambda$ increasing from 0.5 to 30 , and at the highest value the ion flux is reduced by a factor of 0.4 . As in the emission scan, the differences between measured plasma potentials and those from Eq. (11) are approximately the uncertainty in the potential measurements. Figure 5 also shows, for comparison, the model with the charge-exchange correction omitted. The uncorrected 
model differs significantly from the data at pressures above 1 mTorr where the correction for collisions is largest. Although the current of primaries is held constant at $80 \mathrm{~mA}$, there is an increase in the number of secondary electrons as the plasma density is increased.

This increase may be due to the impact of ions, metastable neutrals, or ultraviolet photons releasing additional secondaries from the walls. The temperature found for the secondaries varies with gas pressure indicating that the additional secondaries have an energy distribution different from that of the secondaries from the impact of primary electrons on the walls.

\section{SUMMARY AND CONCLUSION}

A model for the energy balance of confined electrons gives values of plasma potential that are near to measured values for plasmas in a soup-pot type of plasma device. In the model, the confined electrons are heated by equilibration with unconfined secondary electrons from the wall, and the confined electrons lose energy when they pass over the sheath potential barrier at the wall. The energy-balance model requires as input parameters the density $n_{s e}$ and temperature $T_{s e}$ of the secondary electrons, the density $n_{e}$ and temperature $T_{e}$ of the confined electrons, and the mean-free-path for charge exchange collisions $\lambda$. In the experiment, the characteristics of both the confined and the unconfined electrons are found from Langmuir probe data. The ionization rate is found from the plasma parameters using an expression for ion particle balance that includes the effect of charge-exchange collisions on the ion flux to the walls. The calculated and measured values for plasma potential show good agreement over a factor of 8 in filament 
emission current $(20-160 \mathrm{~mA})$, a factor of 64 in pressure $(0.125-8 \mathrm{mTorr})$, and a factor of 30 in electron density $\left(0.4-13 \times 10^{8} \mathrm{~cm}^{-3}\right)$. The differences between the model and the data are comparable to the resolution of the potential measurements, $0.14 \mathrm{~V}$. If the charge-exchange correction to the ion flux is not considered, the model differs from the data by nearly a factor of two at the highest pressure, where the effect of the collisions is the greatest. 


\section{References}

${ }^{1}$ S. Robertson and Z. Sternovsky, "Model for the Density, Temperature, and Plasma Potential of Low-density Hot-Filament Discharges," Phys. Rev. E 72, 016402 (2005).

${ }^{2}$ R. J. Taylor, K. R. MacKenzie, and H. Ikezi, “A Large Plasma Device for Plasma Beam and Wave Studies,” Rev. Sci. Instrum. 43, pp. 1675-1678 (1972).

${ }^{3}$ N. Hershkowitz, R. L. Goettsch, C. Chan, K. Hendricks, and R. T. Carpenter, “Detection of Secondary Electrons in a Multidipole Plasma," J. Appl. Phys. 53, pp. 53305332 (1982).

${ }^{4}$ M.-H. Cho, N. Hershkowitz, and T. Intrator, "Particle and Power Balances of HotFilament Discharge Plasmas in a Multidipole Device,” J. Appl. Phys. 67, pp. 3254-3259 (1990).

${ }^{5}$ A. Lang and N. Hershkowitz, "Multidipole Plasma Density,” J. Appl. Phys. 49, pp. 4707-4710 (1978).

${ }^{6}$ N. Hershkowitz and K. N. Leung, "Plasma Electron Heating by Injsection of Low Energy Electrons,” Appl. Phys. Lett. 26, pp. 607-609 (1975).

${ }^{7}$ J. H. Ingold, "Nonequilibrium Positive Column,” Phys. Rev. E 56, pp. 5932-5944 (1997).

${ }^{8}$ L. D. Tsendin, "Electron Kinetics in Non-Uniform Glow Discharge Plasmas," Plasma Sources Sci. Technol. 4, pp. 200-211 (1995).

${ }^{9}$ V. I. Kolobov and V. A. Godyak, "Nonlocal Electron Kinetics in Collisional Gas Discharge Plasmas,” IEEE Trans. Plasma Sci. 23, pp. 503-531 (1995).

${ }^{10}$ S. A. Self, “Asymptotic Plasma and Sheath Representations for Low-Pressure Discharges,” J. Appl. Phys. 36, pp. 456-459 (1965). 
${ }^{11}$ S. A. Self and H. N. Ewald, "Static Theory of a Discharge Column at Intermediate Pressures," Phys. Fluids 9, pp. 2486-2492 (1966).

${ }^{12}$ K.-U. Riemann, "The Bohm Criterion and Sheath Formation,” J. Phys. D: Appl. Phys. 24, pp. 493-518 (1991).

${ }^{13}$ H. Wallschläger, "Kinetic Description of the Positive Column for Medium KnudsenNumbers," Contrib. Plasma Phys. 30, pp. 385-402 (1990).

${ }^{14}$ Z. Sternovsky, "The Effect of Ion-Neutral Collisions on the Weakly Collisional Plasma-Sheath and the Reduction of the Ion flux to the Wall," Plasma Sources Sci. Technol. 14, pp. 32-35 (2005).

${ }^{15}$ C. B. Opal, W. K. Peterson, and E. C. Beaty, "Measurements of Secondary-Electron Spectra Produced by Electron Impact Ionization of a Number of Simple Gases," J. Chem. Phys. 55, pp. 4100-4106 (1971).

${ }^{16}$ H. Brunet and P. Vincent, "Predicted Electron-Transport Coefficients at High E/N Values. I. Hydrogen,” J. Appl. Phys. 50, pp. 4700-4707 (1979).

${ }^{17}$ Z. Sternovsky and S. Robertson, "Langmuir Probe Interpretation for Plasmas with Secondary Electrons from the Wall," Phys. Plasmas 11, pp. 3610-3615 (2004).

${ }^{18}$ I. P. Shkarofsky, T. W. Johnston, and M. P. Bachynski, The Particle Kinetics of Plasmas. Reading, MA: Addison-Wesley, 1966, ch. 7.

${ }^{19}$ K. Miyamoto, Plasma Physics for Nuclear Fusion. Cambridge, MA: MIT Press, 1989, ch. 4.2.

${ }^{20}$ S. Robertson, Z. Sternovsky, and B. Walch, "Reduction of Asymmetry Transport in the Penning Trap,” Phys. Plasmas 11, pp. 1753-1756 (2004). 
${ }^{21}$ H. Mott-Smith, Jr. and I. Langmuir, "The Theory of Collectors in Gasious Discharges," Phys. Rev. 28, pp. 727-763 (1926).

${ }^{22}$ F. F. Chen, in Plasma Diagnostic Techniques. R. H. Huddlestone and S. L. Leonard, Eds. New York: Academic Press, 1965, ch. 4.

${ }^{23}$ N. Hershkowitz, "How Langmuir Probes Work," in Plasma Diagnostics, O. Auciello and D. L. Flamm, Eds. Boston: Academic Press, (1989), ch. 3.

${ }^{24}$ R. Hegerburg, M. T. Elford, and H. R. Skullerud, "The Cross Section for Symmetric Charge Exchange of $\mathrm{Ne}^{+}$in $\mathrm{Ne}$ and $\mathrm{Ar}^{+}$in Ar at Low energies," J. Phys. B: At. Mol. Phys. 15, pp. 797-811 (1982). 


\section{Figure Captions:}

Fig. 1. (A) A sketch of the source distribution $S(E)$ of electrons created by impact ionization of the neutral gas. Electrons created with energy below $q \Phi_{p}$ replenish the confined electrons. (B) A sketch of the electron energy distribution $F(E)$ found from probe measurements.

Fig. 2. The experimental apparatus. Grids cover the filaments to make the boundary conditions more uniform.

Fig. 3. Plot of the probe current as a function of probe voltage. The modeled ion current has been subtracted. The two vertical lines indicate the probe voltage when at the wall potential and at the plasma potential.

Fig. 4. Plasma parameters from the Langmuir probe measurements with the emission current varied. In the uppermost graph, line segments connect the points for the model function $\Phi_{\mathrm{p}}\left(n_{s e}, T_{s e}, T_{e}, \lambda\right)$. For each set of conditions, three probe sweeps are made, analyzed, and the parameters are averaged. Repeated sweeps closely spaced in time with the same plasma conditions indicate that the uncertainty in the densities and temperatures are less than $10 \%$, which is the uncertainty indicated by the error bars. The error from the probe analysis does not include systematic error. The errors in the model values are smaller than those in the measurements on which they are based as a consequence of the square-root dependencies in Eq. (11). 
Fig. 5. Plasma parameters found from probe measurements with the gas pressure varied. In the uppermost graph, the line segments connect the points for the function $\Phi_{\mathrm{p}}\left(n_{s e}, T_{s e}\right.$, $\left.T_{e}, \lambda\right)$. The function $\Phi_{\mathrm{p}}\left(n_{s e}, T_{s e}, T_{e}, \lambda=\infty\right)$ that omits the charge-exchange correction is also plotted. 

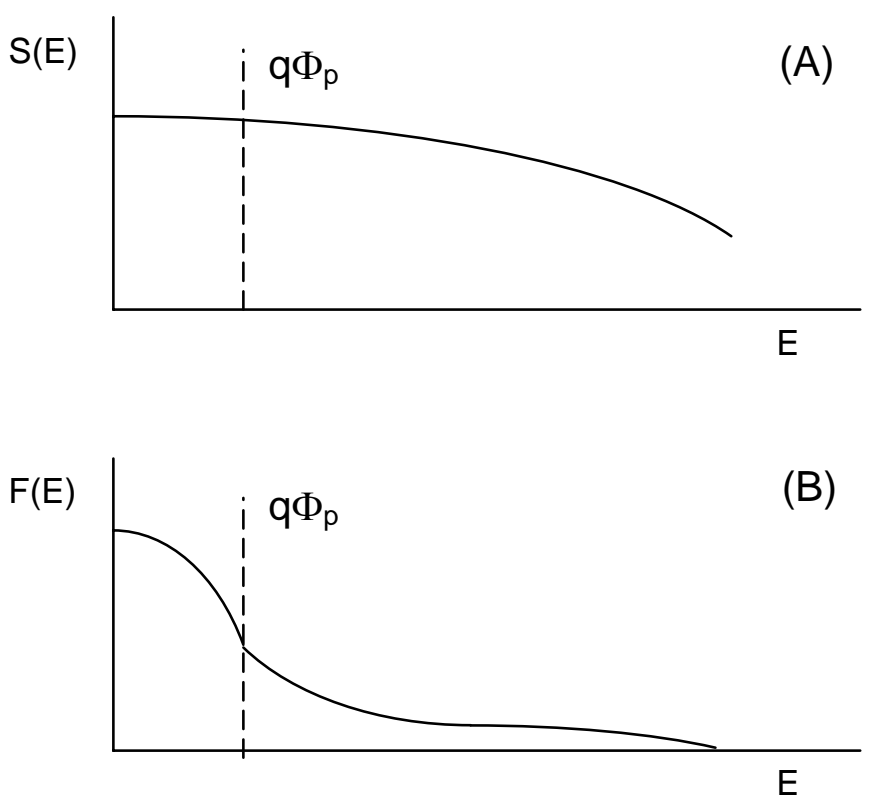

Figure 1

Robertson, Knappmiller and Sternovsky 


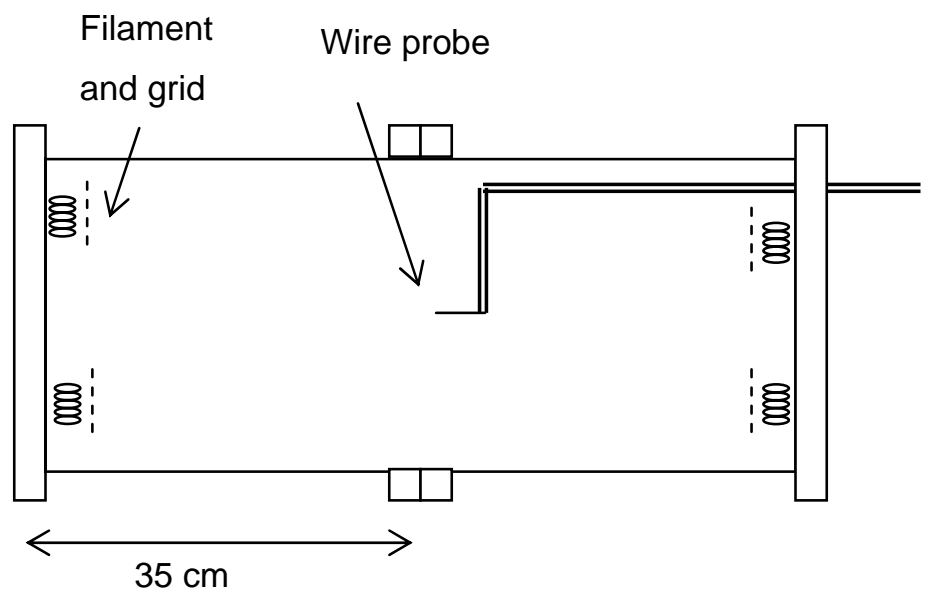

Figure 2

Robertson, Knappmiller and Sternovsky 


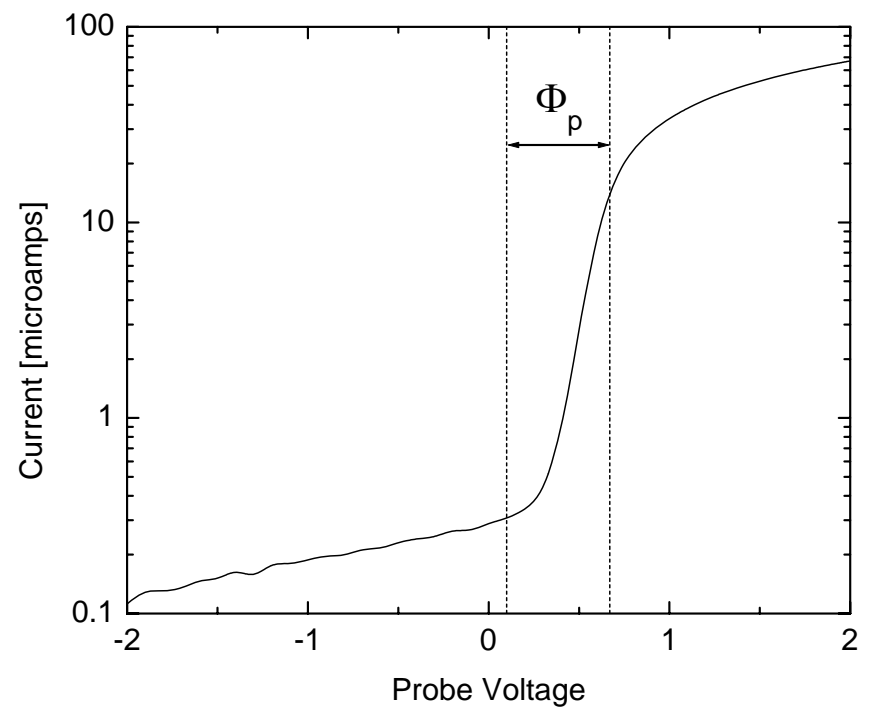

Figure 3

Robertson, Knappmiller and Sternovsky 

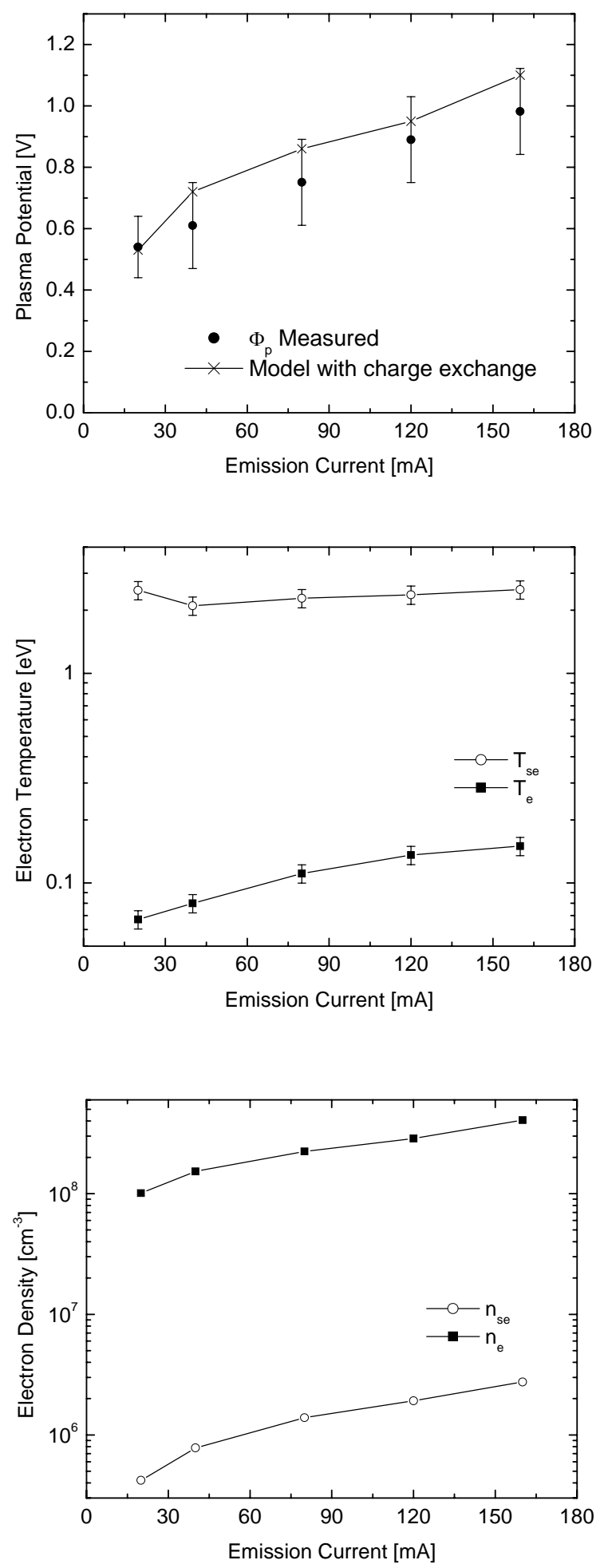

Figure 4

Robertson, Knappmiller and Sternovsky 

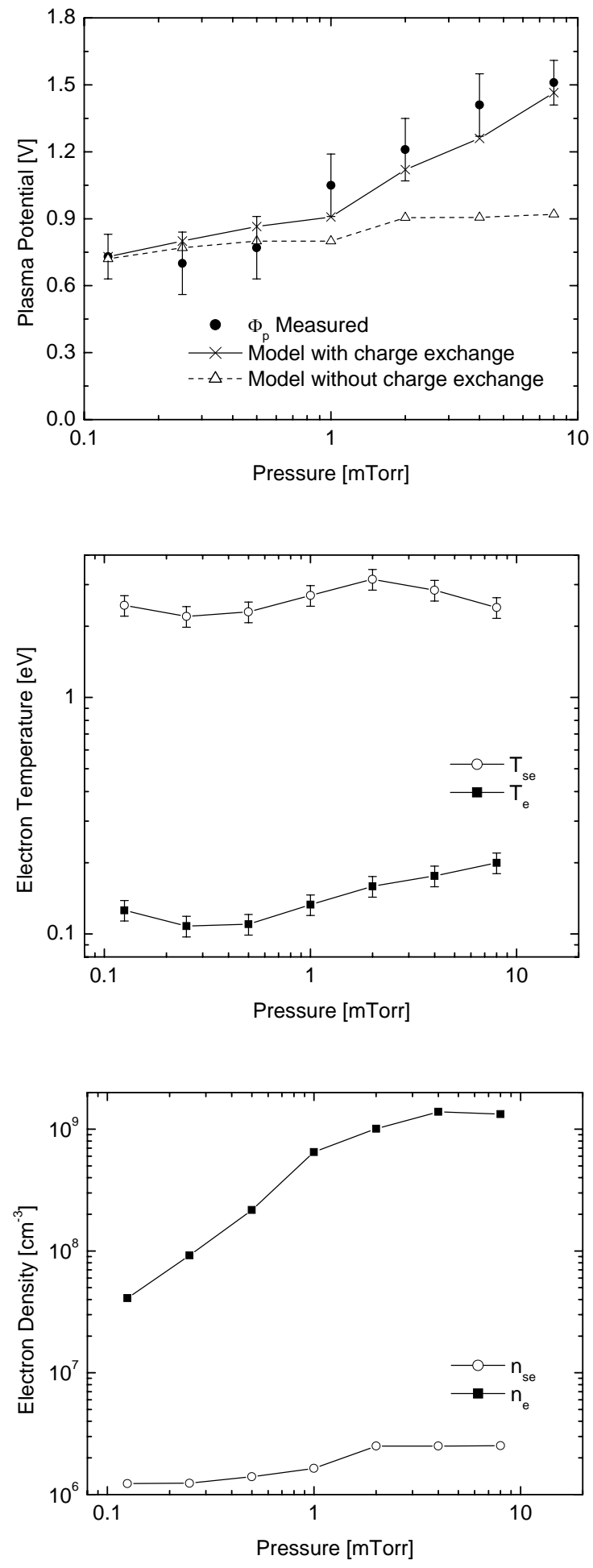

Figure 5

Robertson, Knappmiller and Sternovsky 


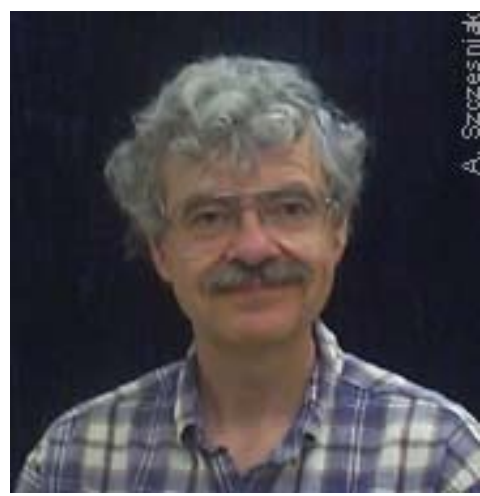

Scott Robertson (M'79-SM'80) was born in Washington, D.C., in 1945. He received B.S. and Ph.D. degrees in applied and engineering physics from Cornell University, Ithaca, N.Y., in 1968 and 1972, respectively. In 1973 he became a Research Associate at Columbia University in New York City where he worked on reflection and focusing of MHD shock waves. In 1975, he moved to the University of California, Irvine, CA, where he became an Associate Research Physicist and worked on collective effects in the propagation and focusing of intense pulsed electron and ion beams. In 1982, he moved to the

University of Colorado at Boulder, CO, where he is now Professor of Physics and Director of the Center for Integrated Plasma Studies. His current research interests are in dusty plasma, ionospheric plasma, and basic plasma physics.

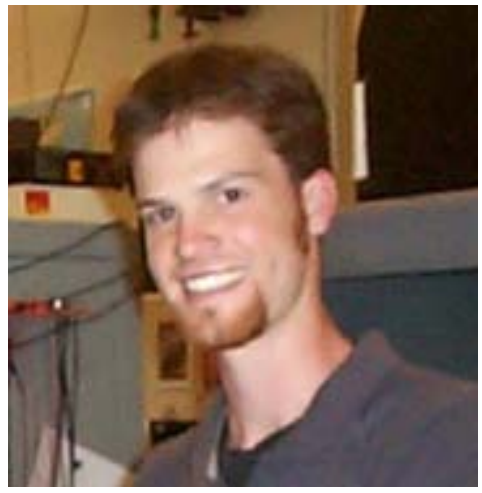

Scott Knappmiller was born in Albany, GA, in 1981. He received a B.S. degree in engineering physics and a B.A. degree in mathematics at the University of Colorado, Boulder, CO, in 2004. He is a Professional Research Assistant at the Center for Integrated Plasma Studies. His interests are in plasma diagnostic methods and instrument development for sounding rockets and spacecraft.

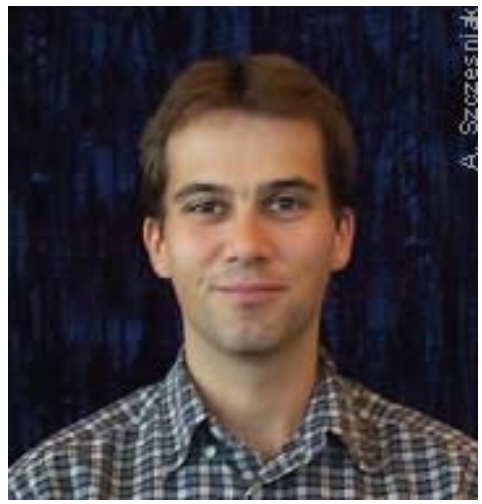

Zoltan Sternovsky was born in Nove Zamky, Slovakia, in 1974. He received M.S. and Ph.D. Degrees in physics from Charles University, Prague, in 1998 and 2001, respectively. He became a Research Assistant at the Center for Integrated Plasma Studies at the University of Colorado in 1999 and became a Research Associate in 2002. He moved to the Laboratory for Atmospheric and Space Physics in 2005. His interests are in ionospheric and space physics, basic plasma diagnostics, and in instrument development for sounding rockets and spacecraft. 